\title{
Human cytomegalovirus infection in women of childbearing age throughout Fars Province - Iran: a population-based cohort study
}

\author{
Arabpour, M. ${ }^{{ }^{*}}$ Kaviyanee, $\mathrm{K} .{ }^{1}{ }^{\text {Jankhah, }}$ A. $^{2}$ and Yaghobi, R. ${ }^{3}$ \\ ${ }^{1}$ Medical Lab, Pars private Hospital, Shiraz, Iran. \\ ${ }^{2}$ Health Genetic Consulting Center, Shiraz, Iran. \\ ${ }^{3}$ Shiraz Transplant Research Center, Shiraz University of Medical Sciences, Shiraz, Iran. \\ E-mail: arabpour@sums.ac.ir
}

\begin{abstract}
Human cytomegalovirus (hCMV) has been described as an important etiological agent of intrauterine infection in women of childbearing age that causes congenital malformation. In the present study we examined 844 serum samples from women of child-bearing age for the presence of IgM and IgG antibodies against hCMV by Elisa technique. 764 out of $844(93 \%)$ of the cases were seropositive for hCMV-IgG and $45(5.4 \%)$ cases were seropositive for hCMV-lgM. An increase in the rate of IgG seroprevalance was associated with an increase in age and parity. The IgG seroprevalance rate was inversely proportional to increasing abortions. Intrestingly seasonal variation affected IgG seroprevalance. There was an increasing trend in IgM positivity rate with age in women less than 29 years. hCMV seroprevalence rate was higher in women from rural as compared to those of urban areas. Finally hCMV primary infections occured in $2.4 \%$ of all pregnancis and it is estimated that up to $0.3 \%$ of all congenital disorders, through out Fars province, were due to hCMV. We suggest a role of child to mother hCMV transmission and sexual maturity as the most probable epidemiological factors of hCMV seroprevalence among women of child bearing age.
\end{abstract}

Keywords: Human Cytomegalovirus, Seroprevalence, IgM and IgG antibodies

\section{INTRODUCTION}

Human cytomegalovirus (hCMV) seroprevalence varies in different populations and age groups. Congenital malformation is the most resulting clinical symptom of hCMV intrauterine infection (Stagno et al., 1986; Demler, 1991; Brooks et al., 2001; Gaytant et al., 2002). Up to $15 \%$ of intrauterine hCMV infections led to congenital symptomatic diseases. Asymptomatic congenital hCMV infection will develop in 10-15\% of infants (Boppana et al., 1992; Fowler et al., 1997; Dahle et al., 2000). Intra uterus transmission of hCMV can occur during primary maternal infection, reactivation, or reinfection of seropositive mothers. Primary hCMV infections are transmitted more frequently to the fetus and are more likely to cause fetal damage than recurrent infections (Stagno et al., 1986; Boppana et al., 1992; Fowler et al., 1992; Fowler et al., 1997; Dahle et al., 2000). If strategies for the prevention of hCMV disease are to be successfully developed, knowledge regarding the epidemiology of the virus is prerequisite. However, a number of hCMV features, including endemic world wide infection, complex natural history, unusual relationship with infected host, ubiquity of infection, and lack of clinical symptoms in most cases, complicate the understanding of the epidemiology of hCMV infection (Forbes, 1989). In this study we examined the association of hCMV seroprevalence with some epidemiological risk factors in women of childbearing age.

\section{MATERIALS AND METHODS}

During 2001 to 2005 serum samples from 844 women at child bearing age (15 to 44 years old) that belonged to different geographical regions of Fars province were collected and stored at $-20{ }^{\circ} \mathrm{C}$ until usage every week. The samples were screened for Specific $\lg M$ and IgG against hCMV antibodies according to manufacturer's instruction (Trinity Biotech co). The results were then interpreted on the basis of antibody titers as seropositive, seronegative and equivocal. Patients with positive IgM were checked for seroconversion two week later. Among patients that were positive for IgM only cases with seroconversion or high IgM titer (Prince et al., 2002) were considered as primary hCMV infections as previously described (Revello and Gerna, 2002). Patients that were simultaneously negative for IgM and IgG antibodies considered as susceptible to hCMV primary infection; whereas those with positive IgG and negative IgM were considered as resistant. Beside serological screening tests for hCMV; total and differential counting of white blood cells (WBCs) was carried out for each case for further analysis.

\section{RESULTS}

\section{hCMV IgM seroprevalence}

45 out of 844 (5.4\%) cases were seropositive for hCMVIgM antibodies. Among positive IgM cases $35.6 \%$ (Pal et al., 1972) were from Shiraz urban area; whereas $4.4 \%$

*Corresponding author 
(Demler, 1991) were from rural areas, the rest of them belonged to other cities of Fars province.

Statistical analysis showed that there was no significant linear correlation (Pearson Correlation) between increasing in $\operatorname{lgM}$ seroprevalence and increasing in age $(p=0.65)$, increasing congenital disorders $(p=0.25)$ and increasing abortions $(p=0.65)$. On the other hand increasing in $\operatorname{lgM}$ seroprevalence associated with increasing gestations and parity numbers $(p<.05)$.

Maximum IgM seroprevalence rate occurred in women between 25-29 years old (Figure 1c). Further statistical analysis revealed that there was no significant linear correlation (Pearson Correlation test) between an increase in age and an increase in hMPV infection rate; although the rate of hCMV infection showed a strong correlation with age group 15-29 years $(p=0.04)$, versus age group29-39 years $(p=0.27)$. More over a soar in IgM seroprevalence associated with age until 29 years.
IgM seroprevalence rate was more in women from rural as compared to those of urban areas. In addition IgM seroprevalence rate was higher in married women in comparison with those of single ones, yet these findings were not statistically significant.

Data indicated that there was significant difference between hCMV-IgM positive rate in pregnant (in 9th Week or more of their gestations) versus non-pregnant women in favor of pregnant women $(p=0.03)$.

$2.3 \%$ (Revello and Gerna, 2002) of all cases had primary or acute hCMV infections. Among pregnant women $2.4 \%$ had primary infections. Also it was revealed that maximum primary infections rate belonged to women between $20-29$ years. Primary infections rate was higher in women from urban as compared to those of rural areas; whereas the reverse was true for recurrent or reinfections rate (Table1). Demographic data and hematological indexes association with the presence of hCMV primary infection are reported in Table 1.

Figure 1: $\mathrm{HCMV} \operatorname{IgM}(\mathrm{a}) \& \mathrm{IgG(b)}$ sero prevalance during four consecutive years througho ut Fars state $\mathrm{HCMV} \operatorname{IgM}(\mathrm{c}) \&$ $\mathrm{IgG}$ (d) sero prevalance in different age groups throughout three differe nr geographical district (Dates are in A.D).
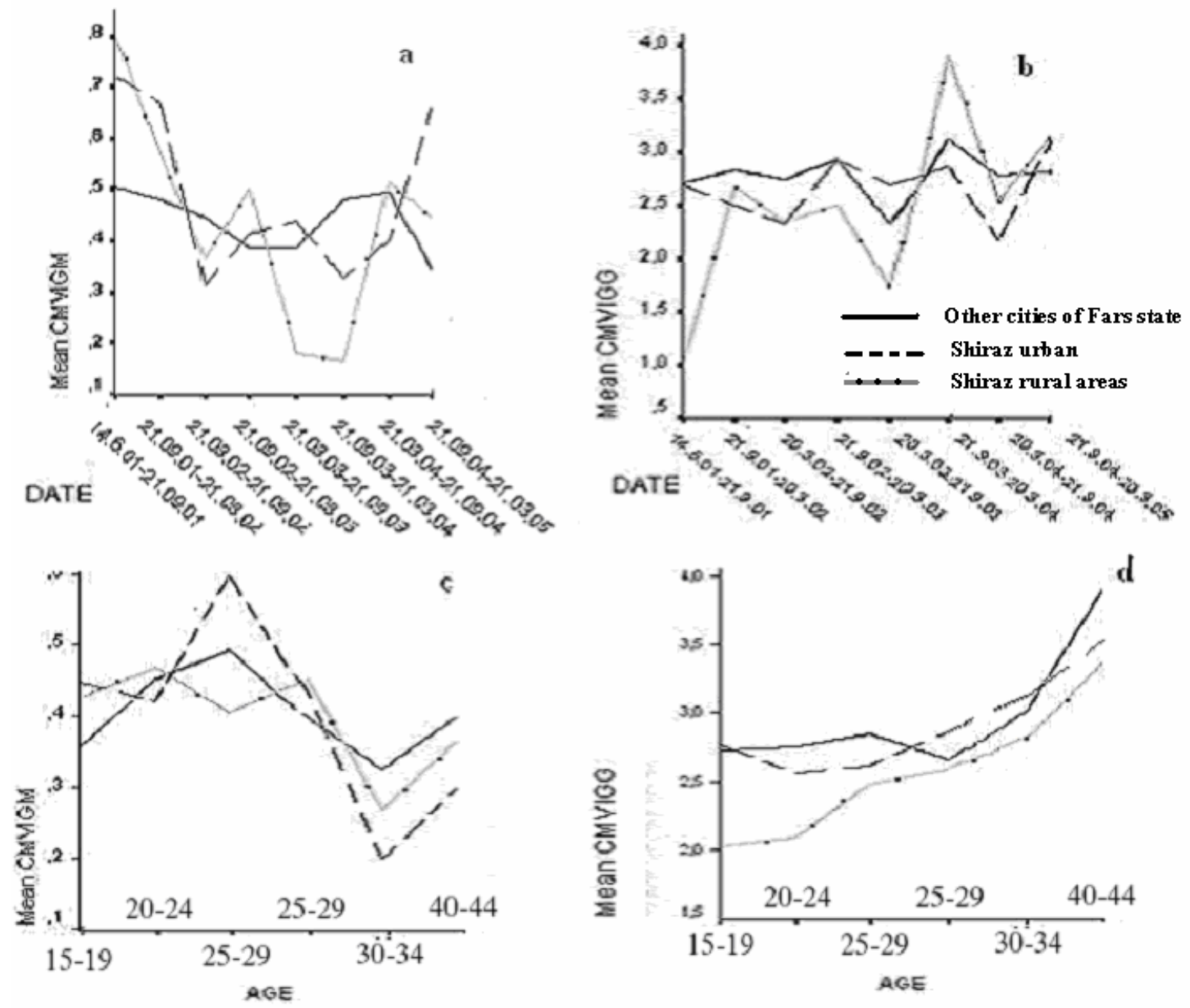
Table 1: demographic characteristic \& hematological indexes distribution of childbearing age women with Primary HCMV infection disease.

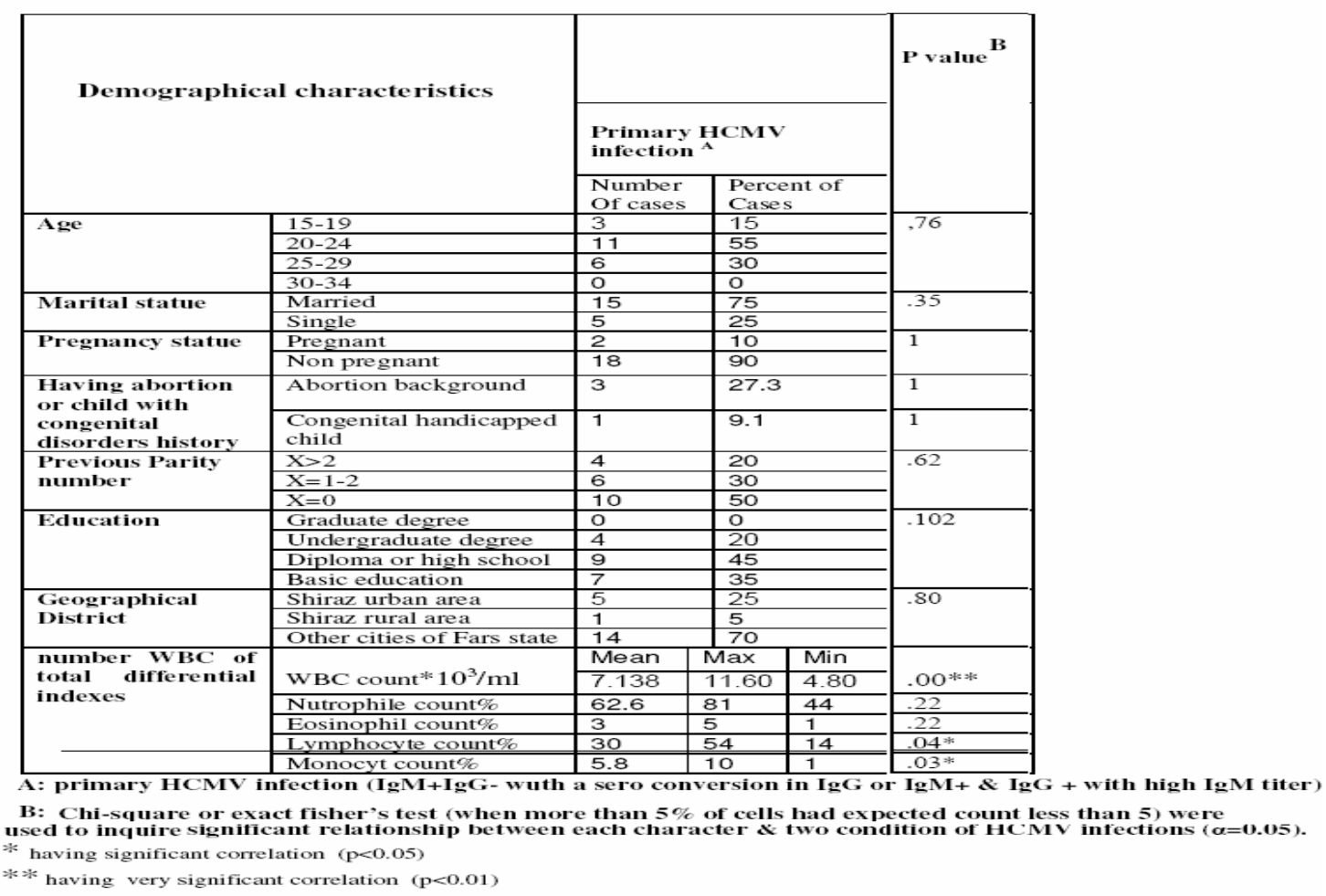

\section{hCMV IgG seroprevalence}

764 (92.9\%) out of 844 cases were seropositive for hCMV-IgG antibodies. Statistical analysis revealed that congenital disorders $(p=0.67)$ wasn't associated with hCMV-IgG seropositivity. On the other hand there was an increasing trend in IgG seropositivity rate with age, increasing parity $(p<001)$, increasing gestations and decreasing abortion $(p=0.03)$. Age distribution of hCMVIgG positive women are reported in Figure1d.

Geographical distribution of IgG positive women was stable throughout Fars province, with almost no statistical difference $(p=0.12)$. An analysis by two tailed independent $\mathrm{T}$ test showed hCMV-IgG prevalence rate among pregnant and non-pregnant patients was almost similar $(p=0.1)$. Also there was no correlation $(p=0.1)$ between marital statue and the rate of IgG seroprevalence.

Surprisingly statistical analysis during five years revealed that unlike IgM, IgG seroprevalence had a vigorous correlation $(p<0.001)$ with seasonal fluctuation especially in mothers: An increase in IgG positivity during fall/winter, versus a decrease during spring/summer (Figure 1a, 1b).

$84 \%(708 / 844)$ of cases were resistant, while $5.5 \%$ (47/844) were susceptible to hCMV primary infections. A comparative correlation of two hCMV-IgG serogroups with hematological indexes and epidemiological risk factors is reported in Table 2.

\section{DISCUSSION}

The prevalence of hCMV antibodies during childbearing age is variable in the world. IgG prevalence is between $40 \%$ to $100 \%$; while IgM seroprevalence rate is between $0 \%$ to $10 \%$, depending on the variability of viral accessibility and its circulation rate in the community (Ray and Mahajan,1997; Mustakangas et al., 2000; Turbadkar et al., 2000; Brooks et al., 2001; Munro et al., 2005). As previously mentioned, our study indicated that IgG seroprevalence rate was $93.0 \%$ that is comparable with those of developing countries (90\% - 100\%) (Brooks et al., 2001; Munro et al., 2005). In contrast, IgM seroprevalence rate $5.4 \%$ is very close to that reported in developed countries (3\%-5.5\%) (Ray and Mahajan,1997; Mustakangas et al., 2000; Turbadkar et al., 2000; Munro et al., 2005).These findings indicated the fact that by the age of 44 almost $93 \%$ of women had encountered hCMV, while active infection due to viral circulation occurred in $5.4 \%$ of the cases during child-bearing age. According to previous studies (Stagno et al., 1986) it is estimated that up to $1 \%$ of fetuses in pregnant women had been infected by hCMV via intrauterine infections, of whom $30 \%$ subsequently developed into congenital diseases. 
As previously described, several epidemiological factors including age, parity numbers, number of gestations, geographical distribution, socioeconomic provinceus, marital provinceus and sexual maturity may contribute to hCMV seroprevalence in women of child bearing age (Pal et al., 1972; Gambarroto et al., 1977; Mathur et al., 1981; Venkitaraman et al., 1986; Griffiths et al., 1991; Wen et al., 1996; De Jong et al., 1998; Gratacap et al., 1998; Hizel et al., 1999).

Age is a determinant factor influencing hCMV seroprevalence. Data indicated that there was an increasing trend in IgG seropositivity rate with age that is in complete concordance with previous findings (Gambarroto et al., 1977; Mathur et al., 1981; Venkitaraman et al., 1986; Gratacap et al., 1998). Also age group 20-29 was determined as the major age group for the occurrence of hCMV primary infections (2.8\%). Further analysis revealed that most marriages (60\%) occurred among the recent age group. According to other studies this data raises the probable role of sexual maturity as a determinative factor of hCMV infections (Forbes, 1989; Brooks et al., 2001).

Table 2: demographic characteristic distribution of childbearing age women within two form of HCMV IgG seroprevalance.

\begin{tabular}{|c|c|c|c|c|c|c|c|}
\hline & & \multicolumn{4}{|c|}{ IgG seroprevalance Statue } & \multicolumn{2}{|c|}{$P$ value ${ }^{A}$} \\
\hline \multirow{2}{*}{\multicolumn{2}{|c|}{ Personal characteristics }} & \multicolumn{2}{|c|}{$\begin{array}{l}\text { Susceptible to Primary } \\
\text { HCMV (IgM- \& IgG -) }\end{array}$} & \multicolumn{2}{|c|}{$\begin{array}{l}\text { Resistant to Primary }{ }^{\text {B }} \\
\text { HCMV (IgM- \& IgG +) }\end{array}$} & \multirow{2}{*}{$\begin{array}{l}\text { IgM- } \\
\text { IgG - }\end{array}$} & \multirow{2}{*}{$\begin{array}{l}\text { IgM- } \\
\text { IgG+ }\end{array}$} \\
\hline & & $\begin{array}{l}\text { Number } \\
\text { Of cases }\end{array}$ & $\begin{array}{l}\text { Percent of } \\
\text { Cases }\end{array}$ & $\begin{array}{l}\text { Number } \\
\text { Of cases }\end{array}$ & $\begin{array}{l}\text { Percent of } \\
\text { Cases }\end{array}$ & & \\
\hline \multirow[t]{6}{*}{ Age } & $15-19$ & 11 & 23.4 & 131 & 18.5 & \multirow[t]{6}{*}{.73} & \multirow[t]{6}{*}{.94} \\
\hline & $20-24$ & 21 & 44.7 & 343 & 48.4 & & \\
\hline & $25-29$ & 11 & 23.4 & 159 & 22.5 & & \\
\hline & $30-34$ & 3 & 6.4 & 56 & 7.9 & & \\
\hline & $35-39$ & 1 & 2.1 & 15 & 2.1 & & \\
\hline & $40-44$ & 0 & 0 & 4 & .6 & & \\
\hline \multirow[t]{2}{*}{ Marital statue } & Married & 24 & 51 & 451 & 63 & \multirow[t]{2}{*}{.08} & \multirow[t]{2}{*}{.07} \\
\hline & Single & 23 & 49 & 257 & 36 & & \\
\hline \multirow[t]{2}{*}{ Pregnancy statue } & Pregnant & 43 & 91.5 & 74 & 10 & \multirow[t]{2}{*}{1.00} & \multirow[t]{2}{*}{.50} \\
\hline & Non pregnant & 4 & 8.5 & 634 & 89 & & \\
\hline \multirow{2}{*}{$\begin{array}{l}\text { Having abortion } \\
\text { or child with } \\
\text { congenital } \\
\text { disorder history }\end{array}$} & Abortion background & 10 & 21.3 & 177 & 25 & $.02 *$ & $.005^{* * *}$ \\
\hline & $\begin{array}{l}\text { Congenital handicapped } \\
\text { child }\end{array}$ & 6 & 12.8 & 80 & 11.3 & .64 & 1.00 \\
\hline \multirow{3}{*}{$\begin{array}{l}\text { Previous Parity } \\
\text { number }\end{array}$} & $\mathrm{X}>2$ & 11 & 23.3 & 96 & 13.5 & \multirow[t]{3}{*}{$.02^{*}$} & \multirow[t]{3}{*}{.19} \\
\hline & $X=1-2$ & 7 & 15 & 257 & 36.3 & & \\
\hline & $\mathrm{X}=0$ & 29 & 61.8 & 355 & 50 & & \\
\hline \multirow[t]{5}{*}{ Education } & Graduate \& higher & 0 & 0 & 4 & .6 & \multirow[t]{5}{*}{.6} & \multirow[t]{5}{*}{.96} \\
\hline & Undergraduate & 10 & 21.3 & 148 & 20.9 & & \\
\hline & Diploma or high school & 19 & 40.4 & 341 & 48.2 & & \\
\hline & Basic education & 18 & 38.3 & 204 & 28.8 & & \\
\hline & Illiterate & 0 & 0 & 11 & 1.6 & & \\
\hline \multirow{3}{*}{$\begin{array}{l}\text { Geographical } \\
\text { District }\end{array}$} & Shiraz urban area & 19 & 40.4 & 233 & 32.9 & \multirow[t]{3}{*}{$.01^{*}$} & \multirow[t]{3}{*}{.05} \\
\hline & Shiraz rural area & 7 & 14.9 & 38 & 5.4 & & \\
\hline & Other cities of Fars state & 21 & 44.7 & 437 & 61.7 & & \\
\hline
\end{tabular}

A : Chi-square or exact fisher's test (when more than $5 \%$ of cells had expected count less than 5 ) were used . $(\alpha=0.05)$

B: data that were suspected to be recurrent infection $(\mathrm{IgM}+, \mathrm{IgG}+)$ \& so logically couldent simultaneously primary infection excluded from this list

* having significant correlation $(\mathrm{p}<0.05)$

*** having very significant correlation $(\mathrm{p}<0.01)$ 
Another factor that may contribute in hCMV infection prevalence is geographical distribution (Wen et al., 1996; Gratacap et al., 1998; Mustakangas et al., 2000; Chakravarty et al., 2005). Unlike previous studies data showed that hCMV-IgG and IgM seroprevalence had no significant correlation, with geographical location, even if hCMV seroprevalence had higher value in rural as compared to those of urban areas. On the other hand primary infections rate was higher through out urban areas. A hypothesis for the probable role of geographical influence upon hCMV seroprevalance might be the route of infection. In rural areas saliva is probably the main route through which the virus is transmitted postnatally. This is likely to be the route through which the virus is transmitted early in life amongst infants and young children due to poor sanitation (Forbes, 1989). On the other hand in urban areas sexual transmission seems to be the major route of infection later in life during childbearing age.

Other factors that may contribute to the hCMV seroprevalence are parity and gestation numbers. In our study perity and gestations numbers were correlated with hCMV seroprevalance.Further analysis with Pearson partial correlation test showed these correlations weren't due to the influence of age interference: the impact of age did not increase enhancing impact of parity ang gestation. These findings are in complete concordance with previous findings (Gaytant et al., 2002; Boppana et al., 1992; Fowler et al., 1992; Gratacap et al., 1998; Turbadkar et al., 2000).

In contrast with previous studies that ruled out the influence of seasonal fluctuation on hCMV seroprevalence (Brooks et al., 2001), hCMV-IgG seroprevalence was higher during fall/winter versus spring/summer. Further analysis revealed the difference is due probably to combinatorial role of day caring centers in transmitting hCMV infection from children to mothers (Forbes, 1989; Gambarroto et al., 1977; Gratacap et al., 1998) and periodical working seasons of day caring centers during fall/winter seasons versus spring /summer: Almost $95 \%$ of mothers population was resistant to hCMV primary infections due to preexisting immunity. Therefore hCMV infections in mother during school opening seasons mostly ended up in IgG immunity boosting, though reinfection where the hCMV strains are different cannot be ruled out (Fowler et al., 1992). By the way more is needed to be done for further confirmation.

An interesting finding in our study was negative association between increasing IgG and obsessive abortions. A decrease in abortions might be an indication that anti-hCMV maternal immunity could prevent abortions due to hCMV infections. Since there wasn't any significant correlation between IgG seroprevalence and congenital disorders, hCMV-IgG circulating antibody is only considered significantly protective against abortion versus congenital disorders. In accordance with previous studies (Mustakangas et al., 2000) these findings indicate that maternal antibodies afford substantial protection to the fetus but the protection is imperfect (Fowler et al., 1992; Boppana et al., 1992).

Eventually statistical analysis revealed that increasing in whole blood cells, lymphocyte and Monocyte counts significantly correlated with hCMV infections, even if lymphocyte count only correlated with primary as compared to recurrent or reinfections. These findings suggest that classic hematological patern for infectious mononucleosis could be seen only during primary infections versus recurrent or reinfections.

Considering all the epidemiologic factors that may contribute to hCMV infection among child-bearing age women through out Fars province, we suggest roles of age, parity, seasonal fluctuation, geographical position and sexual maturity as the most probable associated factors, though these factors are not totally independent and should be considered carefully for interrelated associations. Since there is no effective therapeutic and prophylactics strategies against hCMV infections, primary hCMV infections are very challenging health problem during pregnancy period. So more emphasis should be laid for women of childbearing age, including prospective screening program for hCMV infections before pregnancy, limited contact with hCMV infected children during pregnancy and responsible sexual practices. Also for reducing the chance of abortion implications an effective hCMV vaccination program should be concluded.

\section{ACKNOWLEDGEMENT}

With special thank from Dr Elena Percivalle for her critical review of the paper (Servizio di Virologia, IRCCS Policlinico San Matteo and University of Pavia, Via Taramelli 5, 27100 Pavia, Italy) \& Mrs. Koleyni from ILI Shiraz for her contribution in revising English text.

\section{REFERENCES}

Boppana, S.B., Pass, W.J., Britt, S., et al. (1992). Symptomatic congenital cytomegalovirus infection: neonatal morbidity and mortality. Pediatric Infectious Disease Journal 11: 93 - 99.

Brooks, G.F., Butel, J.S. and Morse S.A. (2001). Herpes viruses Cahpter 33 in Jawetz, Melnick and Adelberg's Medical Microbiology 22nd Edn. Lange Medical Books/McGraw-Hill, USA pp. 382 - 386.

Chakravarty, A., Kashyap, B. and Rathi, K. (2005). The seroepidemiological study on cytomegalovirus in women of child-bearing age with special reference to pregnancy and maternal-fetal transmission. Indian $\mathrm{J}$ Pathol Microbiol 48(4):518 - 521.

Dahle, A.J., Fowler, J.D., Wright, S.B., et al. (2000). Longitudinal investigation of hearing disorders in children with congenital cytomegalovirus. J Am Acad Audiol 11: 283 - 290.

De Jong, M.D., Galasso, G.J., Gazzad, B., et al. (1998). Summary of the II International symposium on 
cytomegalovirus. Antiviral Research 39(3): 141 162.

Demmler, G.J. (1991). Infectious Diseases Society of America and Centers for Disease Control: summary of a workshop on surveillance for congenital cytomegalovirus disease. Infect Dis Rev 13: 315 329.

Forbes, B.A. (1989). Acquisition of Cytomegalovirus Infection: an Update. Rev Clini Microbial 2: 204 216.

Fowler, K.B.S., Stagno, R.F., Pass, W.J., et al. (1992). The outcome of congenital cytomegalovirus infection in relation to maternal antibody provinceus. New England Journal of Medicine 326: 663 - 667.

Fowler, K.B., McCollister, A.J.P., Dahle, S.M.D., et al. (1997). Progressive and fluctuating sensorineural hearing loss in children with asymptomatic congenital cytomegalovirus. J Infect Pediatr 130: $624-630$.

Gambarroto, K., Ranger, R.S., Aubard, Y., et al. (1977). Primary cytomegalovirus infection and pregnant women: Epidemiological study on 1100 women at limoges. Pathol Biol Paris 45(6): 453 - 461.

Gaytant, M.A., Steegers, B.A., Semmekrot, H.M., et al. (2002). Congenital cytomegalovirus infection: review of the epidemiology and outcome. Obstetrical \& Gynecological Survey 57: 245 - 256.

Gratacap, C.B., Bosson, J.L., Morand, P., et al. (1998). Cytomegalovirus seroprevalence in French pregnant women: Parity and place of birth as major predictive factors. European Journal of Epidemiology 14(2):147 $-152$.

Griffiths, P.D., Babboonian, C., Ruttea, D., et al. (1991). Congenital and maternal cytomegalovirus infections in a London population. $\mathrm{Br} \mathrm{J}$ Obstet Gynaecol 98: 135 - 140.

Hizel, S., Parker, S. and Onde, U. (1999). Seroprevalence of Cytomegalovirus infection among children and females of Ankara, Turkey. Pediatrics International 41(5): 506 - 509.

Mathur, A., Jindal, I. and Chaturvedi, U.C. (1981). A serological study of Cytomegalovirus infection at Lucknow. Indian Journal of Medical Research 73: $678-681$.

Munro, S.C., Hall, B., Whybin, L.R., et al. (2005). Diagnosis of and Screening for Cytomegalovirus Infection in Pregnant Women. Journal of Clinical Microbiology 43(9): 4713 - 4718.

Mustakangas, P., Saran, S. and Ammala, P. (2000). Human cytomegalovirus seroprevalance in three different socioeconomically different urban areas during the first trimester: a population based cohort study. International Journal of Epidemiology 29: 585 $-591$.

Pal, S.R., Chitkara, N.L. and Krech, U. (1972). Sero epidemiology of Cytomegalovirus infection in and around Chandigarh (Northern India). Indian Journal of Medical Research 60: 973 - 300.

Prince, H.E. and Leber, A.L. (2002). IgM Levels Validation of an In-House Assay for Cytomegalovirus
Immunoglobulin G (CMV IgG) Avidity and Relationship of Avidity to CMV. Clinical and Diagnostic Laboratory Immunology 9: 824 - 827.

Ray, K. and Mahajan, M. (1997). Seroprevalence of cytomegalovirus antibodies in patients attending. STD and antenatal clinics. 29(2)

Revello, M.G. and Gerna, G. (2002). Diagnosis and Management of Human Cytomegalovirus Infection in the Mother, Fetus, and Newborn Infant. Clinical Microbiology Reviews 15(4): 680 - 715.

Stagno, S.R.F., Pass, G., Cloud, W.J., et al. (1986) Primary cytomegalovirus infection in pregnancy: incidence, transmission to fetus, and clinical outcome. JAMA-Journal of the American Medical Association 256: 1904 - 1908.

Turbadkar, D., Mathur, M. and Rele, M. (2000). Seroprevalance of torch infection in bad obstetric. Indian J Med Microbiol 21 (2): 108 - 110.

Venkitaraman, A.R., Seigneurin, J.M., Lenoia, G.M., et al. (1986). Infections due to the human herpes viruses in Southern India: A sero epidemiological survey. International Journal of Epidemiology 16(4): $561-566$.

Wen, L., Wu, S. and Lu, S. (1996). The epidemiological study on human cytomegalovirus infection in pregnant women and maternal-fetal transmission in three Chinese metropolis. Chung Hua Fuchar Ko Tsa Chih 31(12): 714 - 717. 